\title{
Chemical synthesis and magnetic properties of dilute magnetic ZnTe:Cr crystals
}

\author{
Lijuan Zhao, Bei Zhang, and Qi Pang \\ Physics Department, Hong Kong University of Science and Technology, Clear Water Bay, \\ Hong Kong, China \\ Shihe Yang \\ Chemistry Department, Hong Kong University of Science and Technology, Clear Water Bay, \\ Hong Kong, China \\ Xixiang Zhang, Weikun Ge, and Jiannong Wang ${ }^{\text {a) }}$ \\ Physics Department, Hong Kong University of Science and Technology, Clear Water Bay, \\ Hong Kong, China
}

(Received 28 April 2006; accepted 13 July 2006; published online 30 August 2006)

\begin{abstract}
$\mathrm{ZnTe}$ and $\mathrm{Cr}$ doped ZnTe crystals have been synthesized using a solvothermal process. The characterizations by $\mathrm{x}$-ray diffraction and transmission electron microscopy show that the synthesized $\mathrm{ZnTe}: \mathrm{Cr}$ crystals have a cubic structure and $\mathrm{Cr}$ is incorporated into $\mathrm{ZnTe}$. The magnetic properties of $\mathrm{ZnTe}: \mathrm{Cr}$ are investigated. A hysteresis is observed in the magnetization versus magnetic field measurements below $10 \mathrm{~K}$. The detailed analyses suggest that the hysteresis possibly originated from the magnetic short-range ordering of $\mathrm{Zn}_{1-x} \mathrm{Cr}_{x} \mathrm{Te}$ compounds in the sample. (C) 2006 American Institute of Physics. [DOI: 10.1063/1.2337561]
\end{abstract}

$\mathrm{ZnTe}$ is an attractive material for various optoelectronic devices, ${ }^{1-4}$ such as green light-emitting diodes, electro-optic field detectors, and solar cells. In recent years, studies of transition metal doped dilute magnetic semiconductor (DMS) ZnTe start to attract a lot of attention as DMSs are promising candidates in spintronics studies and applications. It has been shown theoretically that the ferromagnetic state is more stable in $\mathrm{Cr}$ doped $\mathrm{ZnTe}$ than the spin glass state but is less stable in $\mathrm{Mn}, \mathrm{Fe}$, and Co doped $\mathrm{ZnTe}$ if the concentrations of these transition metals are larger than $5 \% .^{5}$ From the magnetic point of view the $\mathrm{Cr}^{2+}$ ion system represents an intermediate case between typical Brillouin-type paramagnetism (typical for Mn and Co DMSs) and Van Vleck-type paramagnetism (Fe-DMS) ${ }^{6,7}$ The most interesting feature of Cr doped DMS is the observed ferromagnetic $p-d$ exchange interaction in excitonic magnetoreflectance for $\mathrm{ZnCrSe}$, $\mathrm{ZnCrS}^{9}$ and $\mathrm{ZnCrTe}^{10}$ Recent work by Saito et al. on $\mathrm{Zn}_{1-x} \mathrm{Cr}_{x} \mathrm{Te}$ thin films grown by molecular beam epitaxy showed that the ferromagnetic Curie temperature $\left(T_{C}\right)$ was around $15 \mathrm{~K}$ with $x=0.035$ (Refs. 11 and 12) and even at room temperature with $x=0.20{ }^{13,14}$ In addition, Pekarek et al. found that the transition temperature is slightly above $300 \mathrm{~K}$ for the ferromagnetic phase in a bulk $\mathrm{Zn}_{1-x} \mathrm{Cr}_{x} \mathrm{Te}$ crystal grown by vertical Bridgman method. ${ }^{15}$ They ascribed the ferromagnetic signal to a pure $\mathrm{CrTe}$ minority phase. Their more recent work showed that single-crystalline $\mathrm{Zn}_{1-x} \mathrm{Cr}_{x} \mathrm{Te}$ grown by vertical Bridgman method with $x=0.0033$ has a $T_{C}$ above room temperature, which was believed to arise from precipitates of $\mathrm{Zn}_{x} \mathrm{Cr}_{y} \mathrm{Te}_{z}$ within the II-VI DMS host. ${ }^{16} \mathrm{Nev}-$ ertheless, rather few reports of the chemical preparation of ZnTe (Ref. 17) and transition metal doped ZnTe are available. In this work, we synthesized ZnTe and $\mathrm{Cr}$ doped $\mathrm{ZnTe}$ crystals by a simple solvothermal process. X-ray diffraction and transmission electron microscopy results show that the

\footnotetext{
a) Author to whom correspondence should be addressed; electronic mail:
} phjwang@ust.hk synthesized ZnTe:Cr crystals have a cubic structure and $\mathrm{Cr}$ is incorporated into $\mathrm{ZnTe}$. The magnetic properties of $\mathrm{ZnTe}: \mathrm{Cr}$ are depicted by a hysteresis observed in the magnetization versus magnetic field measurements below $10 \mathrm{~K}$.

$\mathrm{ZnTe}$ and $\mathrm{Cr}$ doped ZnTe crystals were synthesized using a solvothermal process. It was carried out by standard air-free techniques. A mixture of diethylzink $(1 \mathrm{mmol}, 1 \mathrm{ml})$ and trioctylphosphine oxide (TOPO) $(3 \mathrm{~g})$ which was added as surfactant during the reaction process was heated in a three neck flask to $300{ }^{\circ} \mathrm{C}$ for $1 \mathrm{~h}$. Then $6 \mathrm{ml}$ telluriumtrioctylphosphine (TOP) solution (containing $1.2 \mathrm{mmol}$, $0.153 \mathrm{~g}$ tellurium powder) was quickly injected into this hot solution. The synthesis was carried out under $\mathrm{N}_{2}$ flow. An aliquot at a given reaction time $(1 \mathrm{~h})$ was taken out from the reaction flask with a small syringe and transferred quickly into a vial with toluene. The products were washed with methanol. After centrifugal separation, we obtained ZnTe powders. Cr doped ZnTe crystals were obtained by the same procedure as described above, except adding $0.1 \mathrm{mmol} \mathrm{CrCl}_{2}$ as the source of $\mathrm{Cr}$ to the mixture of diethylzink and TOPO before heating. The following chemical reaction is assumed to take place during the reaction process:

$$
\mathrm{Zn}\left(\mathrm{C}_{2} \mathrm{H}_{5}\right)_{2}+\mathrm{CrCl}_{2}+\mathrm{Te} \rightarrow \mathrm{ZnTe}: \mathrm{Cr} \text {. }
$$

The structures of the obtained materials were characterized by powder $\mathrm{x}$-ray diffraction (XRD) and transmission electron microscopy (TEM). The $\mathrm{Cr}$ content was determined by an x-ray fluorescence (XRF) spectrometer JEOL JSX3201Z. The magnetic properties were measured using a superconducting quantum interference device magnetometer (MPMS-5s).

A typical XRD spectrum for undoped ZnTe crystals is shown in Fig. 1(a). As can be seen, all of the diffraction peaks observed can be indexed in line with the reported data for the bulk cubic ZnTe lattice (Ref. 18, $a_{0}=6.1026 \AA$ ). The expected diffraction peaks are indicated by the vertical bars on the top $x$ axis and the bar lengths are proportional to 


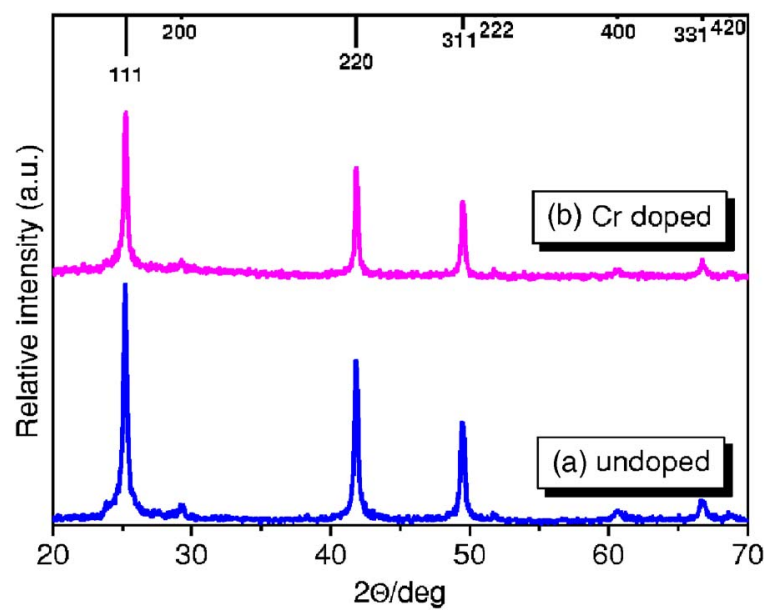

FIG. 1. (Color online) XRD spectra of (a) undoped and (b) Cr doped ZnTe crystals. The upper vertical bars represent the position of the diffraction peaks for the bulk $\mathrm{ZnTe}$ cubic lattice structure and their lengths are proportional to intensity.

intensity. The XRD spectrum of a $\mathrm{Cr}$ doped ZnTe sample is shown in Fig. 1(b). It is evident that no significant difference in the spectrum can be detected in comparison with that of the undoped $\mathrm{ZnTe}$ sample. The $\mathrm{Cr}$ content of about $0.0521 \mathrm{~mol} \%(x \approx 0.00107)$ in the doped sample is determined by the XRF result. At such a low Cr concentration, it is therefore expected that no significant change of the lattice parameter can be induced and the XRD spectra of the undoped and doped samples are identical.

Figure 2 shows the typical TEM images of undoped (a) and $\mathrm{Cr}$ doped (b) ZnTe samples. As it can be seen, the sizes of the synthesized crystals ranged from 100 to $200 \mathrm{~nm}$. The crystal shape is irregular but most of the crystals are single crystalline. Energy dispersive spectrometry analysis indicates no other unexpected impurities in the crystals. It is known that the electron Bohr radius in ZnTe is $\sim 5.2 \mathrm{~nm},{ }^{19}$ which implies that the quantum confinement effect is not observable in our samples.

The measured magnetization curves versus magnetic fields for $\mathrm{Cr}$ doped $\mathrm{ZnTe}$ sample at various temperatures are shown in Fig. 3. The diamagnetic signal due to the host $\mathrm{ZnTe}$ has been subtracted from these curves. A magnified view of the curve measured at $3 \mathrm{~K}$ in the low magnetic field range [Fig. 3(b)] clearly illustrates the existence of a hysteresis loop. This hysteresis loop can be observed up to $10 \mathrm{~K}$. At $25 \mathrm{~K}$ or above, the hysteresis loop disappears.

The temperature-dependent magnetizations were measured in the zero-field-cooled (ZFC) and field-cooled (FC)
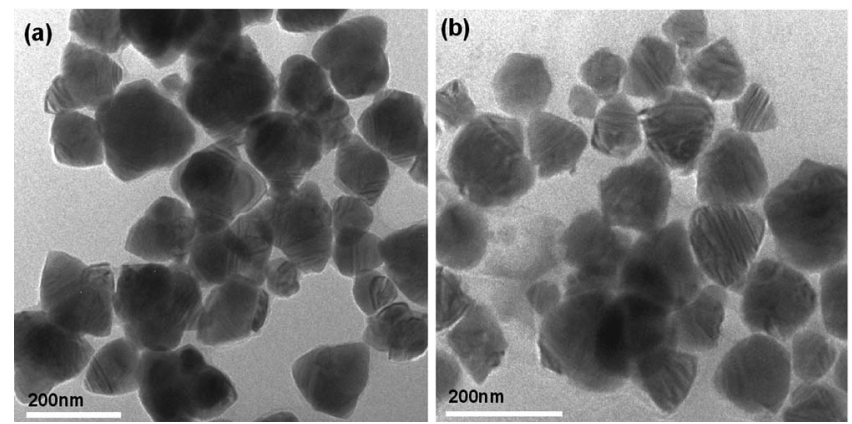

FIG. 2. TEM images of (a) undoped ZnTe and (b) Cr doped ZnTe crystals.

The size of crystals ranges from 100 to $200 \mathrm{~nm}$. temperature for FC magnetization data, the line is a guide to the eyes.
Downloaded 14 Sep 2007 to 143.89 .106 .174 . Redistribution subject to AlP license or copyright, see http://apl.aip.org/apl/copyright.j
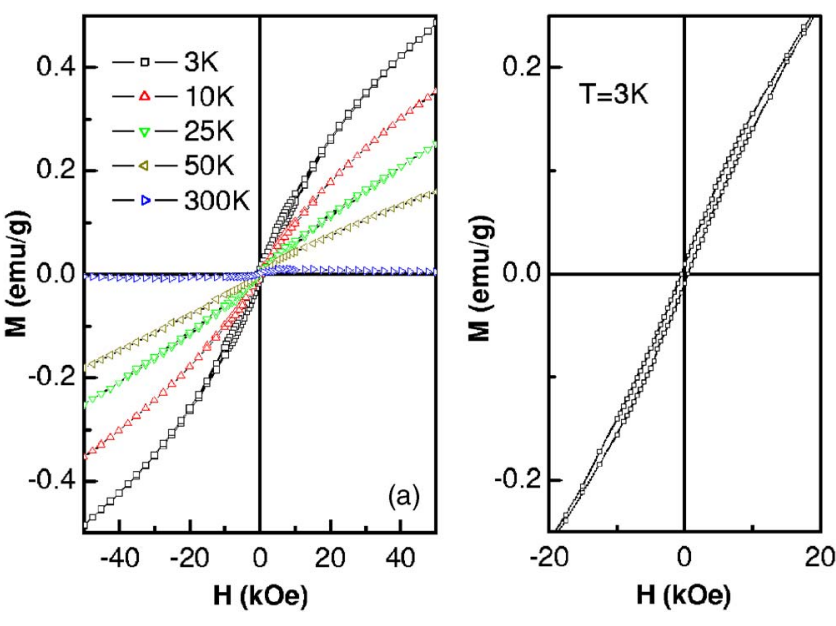

FIG. 3. (Color online) (a) Magnetization vs magnetic field curves for the $\mathrm{Cr}$ doped ZnTe sample measured at various temperatures. (b) A magnified view of the data at $3 \mathrm{~K}$ in low magnetic fields.

processes in a 200 Oe field in the temperature range from 300 to $5 \mathrm{~K}$. Since the sample is purely paramagnetic at high temperatures, only low-temperature data are plotted in Fig. 4 for the sake of clarity. The divergence of the ZFC and FC curves below about $14 \mathrm{~K}$ indicates the existence of metastable states caused either by the blocking of the magnetic moment of the magnetic clusters ${ }^{20}$ or the domain wall pinning. ${ }^{21}$ Since the FC curve monotonically increases with decreasing temperature and follows the Curie-Weiss law below the divergence temperature of $14 \mathrm{~K}$ (see inset of Fig. 4), the magnetic nature of the sample should be ascribed to a magnetic cluster system rather than a bulklike magnet. ${ }^{20,21}$ Due to the blocking of the magnetic clusters, hysteresis loops are expected to appear at temperatures below the divergence temperature, which is in good agreement with the data shown in Fig. 3.

Formation of the magnetic clusters might be due to some precipitates in $\mathrm{Cr}$ doped sample. The most likely magnetic precipitate in $\mathrm{Cr}$ doped sample is reported to be the NiAstype $\mathrm{CrTe}$ compound, whose Curie temperature $T_{C}$ is slightly above $300 \mathrm{~K}^{22}$ For our sample, $\mathrm{CrCl}_{2}$ precipitate is also possible as it is the source of $\mathrm{Cr}$ in the sample preparation process, and $\mathrm{CrCl}_{2}$ is antiferromagnetic with a Néel temperature $\left(T_{N}\right)$ of $16.05 \mathrm{~K}^{23,24}$ If the NiAs-type CrTe compound par-

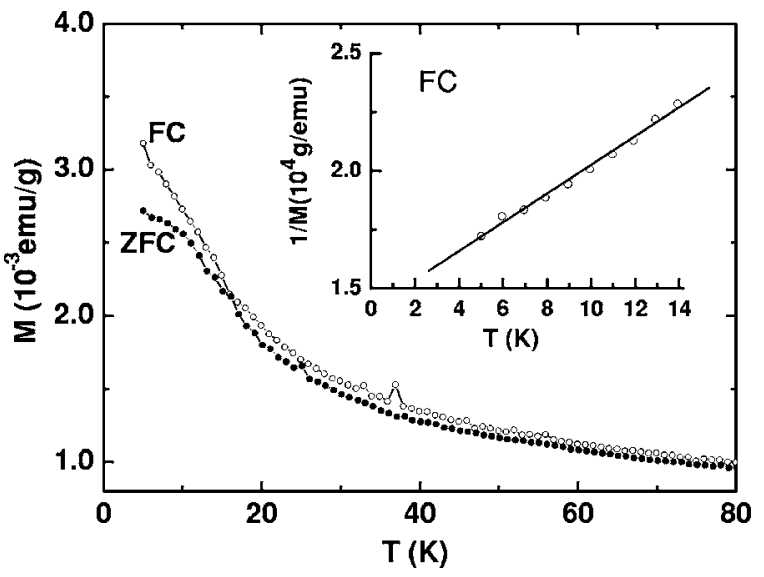

FIG. 4. Temperature dependence of magnetization under zero field cooling and $0.02 \mathrm{~T}$ field cooling for $\mathrm{Cr}$ doped $\mathrm{ZnTe}$. Inset: $1 / M$ as a function of AIP license or copyright, see http://apl.aip.org/apl/copyright.jsp 
ticles or $\mathrm{CrCl}_{2}$ precipitate particles were mixed into the sample, the particles size would be in the range of 100 to $200 \mathrm{~nm}$, as shown in the TEM analysis. Magnetic particles of these sizes generally show a bulk behavior. Therefore, for the ferromagnetic NiAs-type CrTe compound, the $M(T)$ curve will weakly depend on temperature in the low-temperature range and exhibit a sharp drop at its Curie temperature around $300 \mathrm{~K}$, whereas for the antiferromagnetic $\mathrm{CrCl}_{2}$, both ZFC and FC magnetizations will decrease with decreasing temperature below its Néel temperature of $16 \mathrm{~K}$. However, the magnetization curves in Fig. 4 show completely different behaviors, which excludes the possibility that either NiAs-type CrTe compound and/or antiferromagnetic $\mathrm{CrCl}_{2}$ particles are present in the sample.

The remaining possible mechanism responsible for the observed hysteresis and the divergence of low field magnetization curves at low temperatures is then related to shortrange magnetic ordering in $\mathrm{ZnTe}: \mathrm{Cr}$ sample. It has been shown by Saito et al. ${ }^{11}$ that the paramagnetic Curie temperature is significantly higher than the ferromagnetic $T_{C}$ in $\mathrm{Zn}_{x} \mathrm{Cr}_{1-x}$ Te thin film with $x=0.035$. This indicates that the magnetic short-range order remains even far above $T_{C}$. From Fig. 4 , the $T_{C}$ of our sample has not been found but a magnetic short-range order may already exist up to $14 \mathrm{~K}$ as indicated by the obvious hysteresis loops (Fig. 3) and the divergence of the ZFC and FC curves (Fig. 4). But where does the magnetic short-range order originate from? XRF result shows that the $\mathrm{Cr}$ concentration has a quite low value of $x$ $=0.00107$. If the magnetic short-range ordering arises from precipitates of $\mathrm{Zn}_{x} \mathrm{Cr}_{y} \mathrm{Te}_{z}$ due to concentration fluctuations, the Curie temperature $T_{C}$ should be up to room temperature referring to the samples of Pekarek et al. with $x=0.0033 .{ }^{16} \mathrm{It}$ is therefore evident that the precipitates of $\mathrm{Ni}$-As-type CrTe, or $\mathrm{Zn}_{x} \mathrm{Cr}_{y} \mathrm{Te}_{z}$, or $\mathrm{CrCl}_{2}$, or other $\mathrm{Cr}$ ion based compounds are unlikely the origins for explaining the hysteresis loop observed in this sample. As a result, one would expect that a few small ferromagnetic $\mathrm{Zn}_{1-x} \mathrm{Cr}_{x}$ Te clusters might be formed inside the sample because of the low $\mathrm{Cr}$ concentration. These clusters distribute randomly in the ZnTe host and the coupling between them can be ignored for the small number and large separation. The magnetization directions of these clusters are stochastic when there is no external magnetic field, but upon application of a small magnetic field the magnetic moment of those clusters will try to align along the field direction, and moreover, the magnetic moment must overcome the magnetic anisotropy that exists in all magnetic materials to reach their equilibrium. These lead to the divergence between the ZFC and FC magnetization curves at low temperatures. $^{20,25}$ In addition, at low temperature the blocked rotation of the magnetic moment by the anisotropy energy barriers will give rise to the hysteresis loops. ${ }^{20,25}$ It is therefore believed that the short-range magnetic ordering of $\mathrm{Zn}_{x} \mathrm{Cr}_{1-x} \mathrm{Te}$ cluster is responsible for the observed hysteresis loops below $10 \mathrm{~K}$.
In summary, we have synthesized ZnTe and $\mathrm{Cr}$ doped ZnTe crystals using a solvothermal process. XRD spectra of the $\mathrm{Cr}$ doped $\mathrm{ZnTe}$ show no difference from that of the undoped one for very low doping concentration. Magnetic measurements show that hysteresis is observable for $\mathrm{Cr}$ doped sample at and below $10 \mathrm{~K}$. The observed hysteresis signal is believed to arise from the short-range magnetic ordering of $\mathrm{Zn}_{x} \mathrm{Cr}_{1-x} \mathrm{Te}$ clusters.

The authors would like to thank the financial support of the Research Grant Council of Hong Kong SAR via Grant Nos. HKUST6069/02P and 603704. They also thank the MCPF of HKUST for the assistance in the sample characterizations.

${ }^{1}$ B. L. Crower, F. F. Morehead, and P. R. Wagner, Appl. Phys. Lett. 8, 148 (1966).

${ }^{2}$ I. K. Sou, K. S. Wong, Z. Y. Yang, H. Wang, and G. K. L. Wong, Appl. Phys. Lett. 66, 1915 (1995).

${ }^{3}$ Q. Wu, M. Litz, and X. C. Zhang, Appl. Phys. Lett. 68, 2924 (1996).

${ }^{4}$ S. Bhunia and D. N. Bose, J. Cryst. Growth 186, 535 (1998).

${ }^{5}$ K. Sato and H. Katayama-Yoshida, Semicond. Sci. Technol. 17, 367 (2002).

${ }^{6}$ W. Mac, A. Twardowski, H. J. M. Swagten, P. J. Eggenkamp, Y. Shapira, and M. Demianiuk, Phys. Rev. B 50, 14144 (1994).

${ }^{7}$ A. Twardowski, T. Fries, Y. Shapira, P. J. Eggenkamp, H. J. M. Swagten, and M. Demianiuk, J. Appl. Phys. 73, 5745 (1993).

${ }^{8}$ W. Mac, Nguyen The Khoi, A. Twardowski, J. A. Gaj, and M. Demianiuk, Phys. Rev. Lett. 71, 2327 (1993).

${ }^{9}$ W. Mac, Nguyen The Khoi, A. Twardowski, and M. Demianiuk, in The Physics of Semiconductor, Proceedings of the International Conference on the Physics of Semiconductors, Vancouver, Canada, 15-19 August 1994, edited by D. J. Lockwood (World Scientific, Singapore, 1995), p. 2573.

${ }^{10}$ A. Twardowski, Chin. J. Phys. (Taipei) 33, 375 (1995).

${ }^{11}$ H. Saito, W. Zaets, S. Yamagata, Y. Suzuki, and K. Ando, J. Appl. Phys. 91, 8085 (2002).

${ }^{12}$ H. Saito, V. Zayets, S. Yamagata, and K. Ando, Phys. Rev. B 66, 081201R (2002).

${ }^{13}$ H. Saito, V. Zayets, S. Yamagata, and K. Ando, Phys. Rev. Lett. 90, 207202 (2003)

${ }^{14}$ H. Saito, V. Zayets, S. Yamagata, and K. Ando, J. Appl. Phys. 93, 6796 (2003).

${ }^{15}$ T. M. Pekarek, J. E. Luning, I. Miotkowski, and B. C. Crooker, Phys. Rev. B 50, 16914 (1994)

${ }^{16}$ T. M. Pekarek, D. J. Arenas, B. C. Crooker, I. Miotkowski, and A. K. Ramdas, J. Appl. Phys. 95, 7178 (2004).

${ }^{17}$ Y. D. Li, Y. Ding, and Z. Y. Wang, Adv. Mater. (Weinheim, Ger.) 11, 847 (1999).

${ }^{18}$ JCPDS Card No. 15-746 (unpublished).

${ }^{19}$ I. Hernandez-Calderon, in II-VI Semiconductor Materials and Their Applications, edited by M. C. Tamargo (Taylor \& Francis, New York, 2002), p. 125 , and references therein.

${ }^{20}$ X. X. Zhang, J. M. Hernandez, J. Tejada, and R. F. Ziolo, Phys. Rev. B 54, $4101(1996)$

${ }^{21}$ X. X. Zhang, J. M. Hernandez, J. Tejada, R. Sole, and X. Ruiz, Phys. Rev. B 53, 3336 (1996).

${ }^{22}$ T. M. Pekarek, J. E. Luning, I. Miotkowski, and B. C. Crooker, Phys. Rev. B 50, 16914 (1994).

${ }^{23}$ J. W. Stout and R. C. Chisholm, J. Chem. Phys. 36, 979 (1962).

${ }^{24}$ M. Hagiwara and K. Katsumata, J. Magn. Magn. Mater. 140-144, 1665 (1995).

${ }^{25}$ X. X. Zhang, G. H. Wen, Gang Xiao, and Shouheng Sun, J. Magn. Magn. Mater. 261, 21 (2003). 